\title{
Dificultades diagnósticas y terapéuticas en la neurocisticercosis: presentacion de 6 casos y revisión de la literatura
}

\author{
J. Enseñat; R. Martínez-Mañas; J.P. Horcajada*; C. De Juan** y E. Ferrer
}

Servicio de Neurocirugía, Enfermedades Infeciosas* y Neurorradiología**. Hospital Clinic i Provincial. Barcelona.

Resumen

Introducción La neurocisticercosis (NCC) es una patología emergente en países desarrollados debido principalmente, al aumento de la inmigración desde áreas endémicas. El gran poliformismo de la NCC hace necesaria la individualización del tratamiento en cada caso. La toma de decisiones en paises no habituados a esta patología puede ser compleja.

Objetivos. Establecer unas pautas diagnósticas y terapéuticas en los diferentes tipos de NCC.

Material y métodos. Se ha realizado un análisis descriptivo y retrospectivo de seis casos de neurocisticercosis atendidos en el Servicio de Neurocirugía del Hospital Clínic de Barcelona desde 1992 al 2000 (ambos incluidos). Se realiza una revisión de la literatura sobre los métodos diagnósticos y terapéuticos actuales en las diferentes modalidades de NCC.

Discusión. El diagnóstico definitivo o probable de NCC se realiza en función de criterios clínicos, inmunológicos, radiológicos y epidemiológicos. En pacientes con enfermedad inactiva se recomienda únicamente tratamiento sintomático. No hay datos concluyentes sobre el beneficio del tratamiento con antiparasitariosenlaenfermedadactivaparenquimatosa, sin embargo, los pacientes con enfermedad activa extraparenquimatosa se pueden beneficiar del tratamiento antihelminítico asociado a corticoides durante los primeros días. El tratamiento quirúrgico está indicado en lesiones que provocan focalidad neurológica progresiva, hipertensión endocraneal o hidrocefalia.

PALABRAS CLAVE: Neurocisticercosis. Terapia antiparasitaria. Cirugía. EITB. ELISA.

Diagnostic and therapeutic difficulties in neurocysticercosis: presentation of 6 cases and review of the literature

Recibido; 12-04-06. Aceptado: 12-07-06
Summary

Introduction. The incidence of neurocysticercosis (NCC) is increasing currently in developed countries due to the migration movements from endemic countries. Due to NCC polymorphism, treatment would be individualized in each case. Countries not used to this disease have to deal with.

Goals. To set up diagnostic and therapeutic guidelines in all sorts of NCC and choose the correct treatment would be challenging.

Patients and methods. To perform a descriptive and retrospective analysis of six cases of NCC seen in the Neurosurgery Department of the Hospital Clinic de Barcelona from 1992 to 2000 (both included). We have performed a revision of the literature about diagnostic and therapeutic methods.

Discussion. Definitive or probable diagnosis of NCC is based on clinical, imaging, immunological, and epidemiological criteria. In patients with inactive disease only symptomatic treatment is indicated. In active parenchymal forms there are not consensus if antiparasitic treatment is indicated. However, in extraparenchymal active disease aggressive treatment with antiparasitic agents and steroids is recommended. In cases of intracranial hypertension, neurological deficits or hydrocephalus surgery is the treatment of choice.

KEY WORDS: Neurocysticercosis. Antiparisitic drugs. Surgery. EITB. ELISA.

Abreviaturas. AC: anticuerpo. Ag: antígeno. DVP: derivación ventrículo-peritoneal. EITB: electroimmunotransfer Blot. ELISA: enzyme-linked Immunosorbent Assay. HbsAg: antígeno de superficie para hepatitis B. HIV: virus de la inmunodeficiencia humana. HTIC: hipertensión intracraneal. IG: Inmunoglobulina. LCR: líquido céfalo-raquídeo. NCC: neurocisticercosis. RM: resonancia magnética. SN. sistema nervioso. SNC: sistema nervioso central. TC: tomografia computarizada. VHC: virus hepatitis $B$. 


\section{Introducción}

La neurocisticercosis (NCC) es la parasitosis más frecuente del SNC, aunque en la actualidad es una patología poco conocida para muchos neurocirujanos jóvenes. Se estima que en la actualidad hay 50.000 .000 personas infectadas en todo el mundo, aunque conocer su prevalencia es difícil ya que el $49 \%$ de infectados se encuentran asintomáticos ${ }^{3,37}$. La NCC es una patología endémica en países en vías de desarrollo(Méjico,América Central y Sur-exceptoArgentina y Uruguay-, Africa -excepto paises musulmanes-, India, Indonesia, Tailandia y China ), y todavía en algunas zonas de Europa, y está ligada a condiciones sanitarias y de higiene precarias. Los movimientos migratorios y el turismo a zonas endémicas han favorecido su reaparición en países desarrollados ${ }^{19,42}$. Durante los últimos años el número de casos diagnosticados en España ha aumentado de manera notable $14,35,36$.

La principal característica de la NCC es su gran polimorfismo. La gran variedad de patrones de presentación clínico-radiológicos y el número de patologías que mimetiza supone un desafio. El tratamiento de esta entidad es complejo, ya que depende de la localización, estadío evolutivo del parásito y la sintomatología. El abordaje más efectivo para la teniasis y cisticercosis es la prevención, la cual debe ser el objetivo primario de salud pública en paises subdesarrollados. Actualmente, el principal tratamiento son los fármacos antiparasitarios, pero en aquellos casos en que la opción terapéutica es la cirugía se deben evaluar diferentes estrategias, siendo la endoscopia una alternativa quirúrgica en las localizaciones intraventriculares.

El objetivo de este trabajo es presentar una revisión de la NCC desde el punto de vista diagnóstico y terapéutico e intentar establecer unas pautas de tratamiento, ya que se trata de una enfermedad poco habitual en nuestro medio, aunque en aumento, y con unas dificultades de manejo que pueden conducir a la toma de decisiones erróneas.

\section{Pacientes y métodos}

Presentamos un estudio retrospectivo y descriptivo de seis casos de NCC ingresados en el Servicio de Neurocirugía del Hospital Clínic i Provincial de Barcelona durante un periodo de 9 años (1992-2000).

\section{Caso 1}

Mujer de 32 años de nacionalidad peruana y sin antecedentes de interés, que consultó de urgencia por clínica de crisis parciales, en forma de parestesias en el hemicuerpo izquierdo, de 6 meses de evolución, y la aparición de cefalea y vómitos de intensidad creciente durante los últimos 15 días. La exploración neurológica reveló una

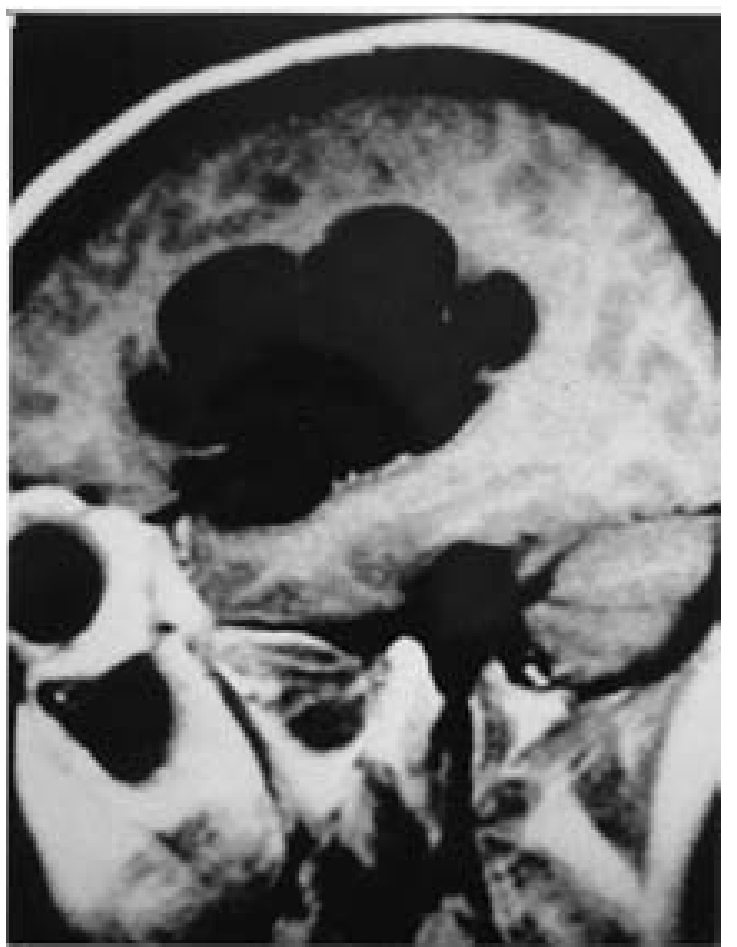

Figura 1. RM sagital potenciada en T1, sin contraste. NCC subaracnoidea: lesión quística de gran tamaño, polilobulada y tabicada, de membranas finas y líquido isointenso con el LCR que ocupa la cisura de Silvio derecha. La lesión ejerce efecto de masa sobre las estructuras adyacentes y deforma la cisura.

hemihipoestesia superficial izquierda y una paresia facial central ipsilateral. La TC y RM craneal (Fig. 1) mostraron una voluminosa lesión córtico-subcortical temporo-parietal derecha, tabicada y calcificada, compatible con NCC racemosa. La serología en sangre y LCR fue positiva para NCC. Se inició un tratamiento con Praziquantel, pero debido a la persistencia del síndrome de hipertensión intracraneal (HTIC) la paciente fue intervenida quirúrgicamente practicando una craneotomía pterional y extirpación de la lesión polivesicular sin incidencias. La TC de control mostró la resección completa de la lesión quística con persistencia de las calcificaciones. La paciente evolucionó favorablemente hallándose asintomática al año de su intervención.

\section{Caso 2}

Paciente de 5 años, de nacionalidad colombiana, traída a urgencias por presentar una crisis parcial motora simple en extremidad superior izquierda. La exploración neurológica post-crítica fue normal. La TC y RM (Fig. 2) mostraron una lesión nodular, sólida, parietal derecha compatible con la forma celulosa de cisticercosis cerebral. El estudio de extensión y serologías en heces, LCR y suero fueron nega- 


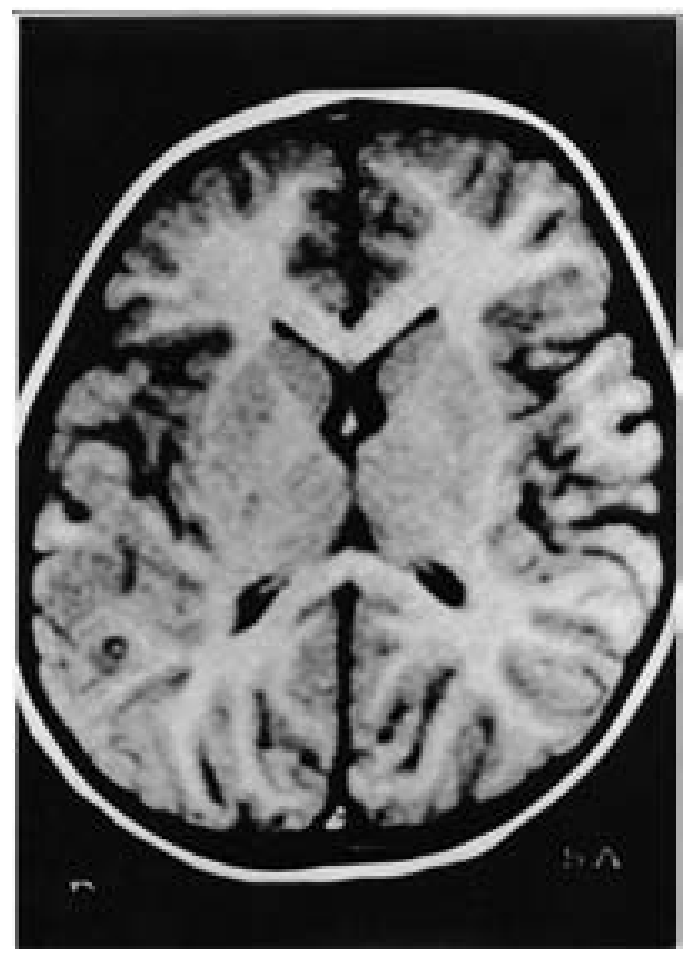

Figura 2. RM axial potenciada en T1 sin contraste. NCC parenquimatosa: lesión nodular corticosubcortical de localización temporal posterior derecha. Imagen en diana con un punto central hiperintenso, que es el escólex y rodeada de un líquido isointenso con el LCR.

tivos. Se inició tratamiento anticomicial con valproato sódico y antiparasitario con albendazol. La paciente no requirió cirugía y actualmente permanece asintomática.

\section{Caso 3}

Mujer de 68 años, natural de Málaga, que consultó por clínica de inestabilidad a la marcha de 2 meses de evolución y deterioro progresivo de funciones superiores. A destacar entre sus antecedentes el diagnóstico de NCC hacía 7 años a raíz de crisis comiciales. A su ingreso la exploración neurológica mostró una paciente desorientada, con alteración de la memoria de fijación, dificultad a la elevación de la mirada conjugada y ataxia de la marcha. La serología para cisticerco fue positiva en plasma y negativa en LCR. La RM craneal mostró múltiples calcificaciones cerebrales, moderada dilatación ventricular y una lesión nodular en la parte superior del IV ventrículo, todo ello compatible con NCC. Se instauró tratamiento antiparasitario con albendazol seguido de la colocación de un drenaje ventricular externo y exéresis de la lesión del IV ventrículo por vía suboccipital. En un segundo tiempo se implantó un sistema de derivación ventrículo-peritoneal debido a la persistencia de la hidrocefalia. La paciente se recuperó completamente y ha permanecido asintomática, con tratamiento anticomicial.

\section{Caso 4}

Mujer de 57 años, procedente de un entorno ganadero de Calabria (Italia), estudiada por un neurólogo a raíz de crisis tónico-clónicas generalizadas. El examen neurológico fue normal. La TC mostró una lesión poliquística, temporal izquierda, isodensa al LCR, con captación periférica de contraste. En la RM el contenido quístico era ligeramente hipointenso respecto al LCR. Fue remitida a nuestro servicio con el presunto diagnóstico de quiste epidermoide de cisura de Silvio, pero tras la revisión de las imágenes radiológicas se evocó la posibilidad de una parasitosis cerebral. Los exámenes séricos complementarios mostraron anticuerpos antiequinococo positivos a índices significativos. Se decidió la exéresis quirúrgica de la lesión a través de craneotomía temporal izquierda. La paciente permaneció asintomática en el postoperatorio y el diagnóstico anatomopatológico confirmó una cisticercosis racemosa en el espacio subaracnoideo. Se completó el tratamiento con albendazol. La TC de control mostró una lesión residual sin signos de actividad que se controla periódicamente.

\section{Caso 5}

Varón de 21 años, procedente del norte de Italia (Marque), residente en Sudamérica, que consultó por crisis comiciales tónico-clónicas generalizadas. La exploración neurológica fue normal. La RM mostró un nódulo frontal derecho con edema perilesional, centro hipodenso y captación periférica de contraste compatible con absceso cerebral. Los exámenes complementarios practicados fueron normales, incluyendo serologías para HIV, VHC, HbsAg. Se instauró tratamiento antibiótico empírico, pero la lesión no se modificó en 4 meses. Se decidió la resección quirúrgica de la lesión a través de una craneotomía guiada por estereotaxia para establecer el diagnóstico. El examen patológico reveló una cisticercosis intraparenquimatosa. La RM de control no mostró lesión residual y el paciente permanece asintomático en el momento actual.

\section{Caso 6}

Mujer de 27 años, de nacionalidad colombiana, que ingresó de urgencia por un cuadro de 12 horas de evolución de cefalea intensa, vómitos y deterioro progresivo del nivel de conciencia. A su llegada la paciente estaba somnolienta, con apertura ocular al dolor y sin déficit motor. La TC evidenció una hidrocefalia supratentorial aguda, por lo que se colocó un drenaje ventricular externo de urgencia. A las 6 horas la paciente se restableció por completo. La 


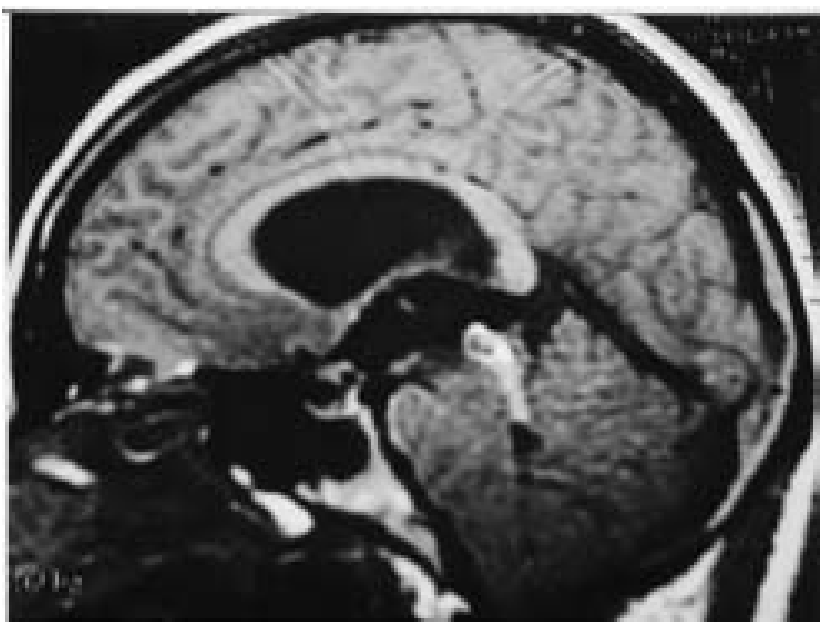

Figura 3. RM sagital potenciada en T1 tras inyección de contraste paramaanético. NCC intraventricular. Lesión heterogénea que capta contraste en periferia, que ocupa la porción posterior del III ventrículo, obstruye el acueducto de Silvio en su totalidad y aflora en el IV ventrículo y provoca una hidrocefalia triventricular secundaria.

RM craneal (Fig. 3) mostró una lesión en el receso posterior del tercer ventrículo, compatible con un coágulo sanguíneo como primera hipótesis. Se decidió practicar una endoscopia cerebral a fin de realizar una ventriculostomía premamilar para tratar la hidrocefalia y exéresis de la lesión para establecer un diagnóstico. Durante el acto quirúrgico se descubrió una lesión quística, lobulada, que obstruía la entrada del Acueducto de Silvio, que se pudo resecar completamente (Fig 4). La TC de control mostró una exéresis completa con resolución de la hidrocefalia. El informe anátomo patológico confirmó una forma racemosa de cisticercosis. Se instauró un tratamiento con albendazol. La paciente permanece asintomática y sin derivación de LCR hasta el momento actual.

\section{Discusión}

La cisticercosis es una parasitosis causada por el enquistamiento de larvas de Taenia solium. El hombre actúa como huésped definitivo, mientras que el cerdo es el huésped intermediario. La fuente de infección en humanos y cerdos es la ingestión de alimentos contaminada con heces humanas de portadores de T. solium, de modo que incluso los vegetarianos estrictos pueden contagiarse. Laingestión de carne de cerdo poco cocinada e infectada con cisticercos es la vía exclusiva para desarrollar la teniasis intestinal, lo cual cierra el ciclo del parásito.

En el hombre, el cisticerco se puede alojar en el SNC (sobre todo sustancia gris, tejido periventricular y leptomeninges), en el sistema músculo-esquelético y en el globo ocular. Presenta una marcada predilección por

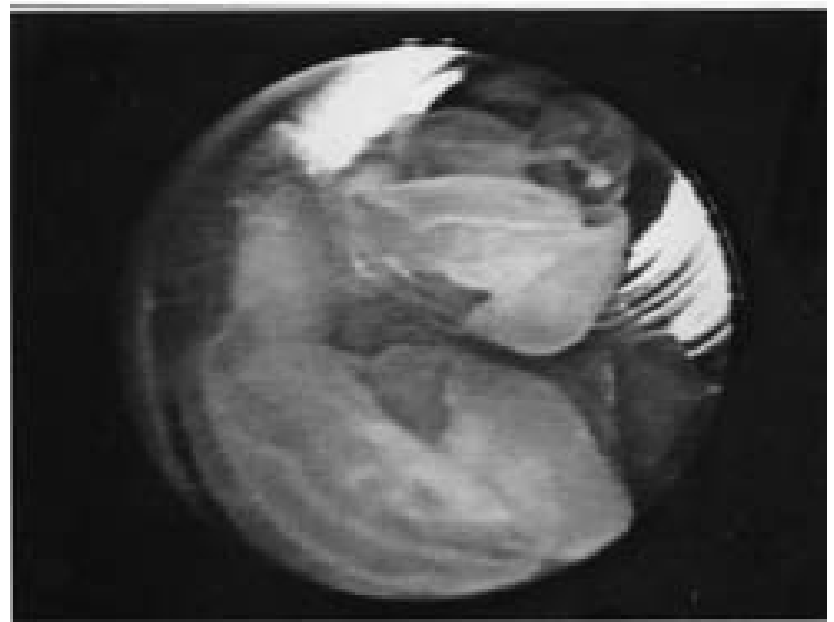

Figura 4. Imagen endoscópica del quiste parasitario. Son características sus paredes finas, blanquecinas y friables, que hacen difícil en ocasiones su exéresis completa.

el cerebro (60-90\% de los casos), mientras que la médula espinal y SN periférico se afectan raramente. En el cerebro humano el cisticerco puede hallarse en el espacio subaracnoideo-meníngeo (más frecuente en la forma racemosa), intraventricular e intraparenquimatoso (típico de la forma celulosa), aunque también existen formas mixtas. Actualmente se están utilizando clasificaciones que valoran el estado activo de la enfermedad. Se habla de formas inactivas cuando no existe evidencia de que el parásito sea viable, o bien cuando el parásito está en proceso de degeneración. Las formas inactivas más frecuentes son las calcificaciones parenquimatosas. La forma activa, o parásito vivo, es bien tolerada por el cerebro humano, de modo que los síntomas aparecen con la muerte del parásito y la subsecuente reacción inflamatoria del SNC.

La NCC suele ser más frecuente en adultos. La forma de presentación más habitual son las convulsiones (en áreas endémicas es la primera causa de epilepsia) ${ }^{13}$, seguido de la hipertensión intracraneal (HTIC), trastornos psiquiátricos y meningitis de repetición. Durante la enfermedad estos síntomas se pueden solapar e incluso asociar. La pérdida de la conciencia asociada a los movimientos de la cabeza (Síndrome de Bruns) se asocia a la cisticercosis del IV ventrículo. La HTIC suele ser secundaria a hidrocefalia por oclusión inflamatoria de los agujeros de Luscka y Magendie. La sintomatología focal aparece en las afectaciones de cisterna cerebelopontina, cisura de Silvio y convexidad. El infarto cerebral es una complicación rara de la NCC subaracnoidea y se produce por la oclusión de pequeñas arterias terminales. También se han descrito casos de aneurismas inflamatorios ${ }^{21}$. La NCC en la región sellar es rara, de difícil diagnóstico antes de la cirugía, y clínicamente simula tumoraciones de esta región. 


\section{Tabla 1}

Criterios diagnósticos de cisticercosis

\begin{tabular}{|c|c|}
\hline & Criterios \\
\hline \multicolumn{2}{|l|}{ Absoluto } \\
\hline$\square$ & Demostración histológica del parásito en una biopsia de una lesión cerebral o medular \\
\hline & Lesiones quísticas que muestren el scolex en la TC o RM \\
\hline$\square$ & Visualización directa de parásitos subretinianos en examen fundoscópico \\
\hline \multicolumn{2}{|l|}{ Mayor } \\
\hline$\square$ & Lesiones altamente sugestivas de NCC en estudios de neuroimagen \\
\hline$\square$ & Suero positivo para la detección de anticuerpos anticisticerco en immunoblot \\
\hline$\square$ & Resolución de lesiones quísticas intracraneales tras tratamiento con albendazol o praziquantel \\
\hline$\square$ & Resolución espontánea de lesiones únicas pequeñas captantes \\
\hline \multicolumn{2}{|l|}{ Menor } \\
\hline$\square$ & Lesiones compatibles con NCC en estudios de neuroimagen \\
\hline$\square$ & Manifestaciones clínicas sugestivas de NCC \\
\hline$\square$ & LCR positivo en ELISA para la detección de anticuerpos anticisticerco o antígenos de cisticerco \\
\hline$\square$ & Cisticercosis fuera del SNC \\
\hline \multicolumn{2}{|c|}{ Epidemiológico } \\
\hline$\square$ & Evidencia de un contacto con un huesped infectado por T. Solium \\
\hline$\square$ & Individuos procedentes de un área donde la cisticercosis es endémica \\
\hline$\square$ & Historia de viajes frecuentes a áreas endémicas \\
\hline
\end{tabular}

TC: tomografía computarizada. RM: resonancia magnética. NCC: neurocisticercosis. LCR: líquido cefalorraquídeo

\section{Diagnóstico}

Todo paciente residente en un área endémica y que presente clínica de meningitis recidivante, o bien sintomatología focal sugestiva de lesiones ocupantes de espacio, debe hacernos sospechar una NCC.

El diagnóstico de NCC es difícil, ya que las manifestaciones clínicas son inespecíficas, los hallazgos radiológicos no son patognomónicos y algunos tests serológicos tienen poca sensibilidad o especificidad. en 1997, se propusieron un grupo de criterios diagnósticos recientemente revisados, basados en aspectos clínicos, radiológicos, inmunológicos y epidemiológicos ${ }^{17}$. Estos criterios proporcionan 2 grados de certeza diagnóstica (tabla I):

- Definitivo: paciente con un criterio absoluto o dos mayores, un menor y un epidemiológico.

- Probable: paciente con un criterio mayor y dos menores, o un mayor, un menor y un epidemiológico, o 3 meno- res y un epidemiológico.

En la NCC, el análisis simple del LCR suele ser inespecífico mostrando pleocitosis linfocítica o eosinofílica con hipoglucorraquia y elevación del nivel de proteínas. El grado de respuesta inflamatoria orienta sobre la reacción del huésped frente al parásito. La presencia de pleocitosis y anticuerpos específicos en el LCR coincide con el estado de degeneración del cisticerco y la intensificación de la respuesta inmunoinflamatoria del huésped ${ }^{41}$.

La NCC tiene un perfil evolutivo muy variable que depende de características inmunológicas. La relación entre huésped y parásito es compleja. Los mecanismos de evasión inmunológica, a parte de diferentes grados de inmunosupresión, permiten una supervivencia mayor y pacífica del parásito en el SNC sin producir reacción inflamatoria significativa. Generalmente, la actividad clínica sucede cuando se inicia la degeneración del quiste. A menudo, coexisten múltiples quistes en diferentes fases 
de evolución en el mismo paciente haciendo mas difícil el manejo clínico. El diagnóstico correcto per se no es suficiente para determinar la gravedad, régimen terapéutico adecuado y pronóstico. Es necesario conocer si la enfermedad es activa: 1, bajo criterios inmunológicos y clínicos y 2. bajo criterios radiológicos (quistes sin captación).

\section{Diagnóstico serológico}

\subsection{Test de ELISA o fijación del complemento en LCR para anticuerpos}

La reacción positiva para los antígenos del cisticerco en el test de ELISA o fijación del complemento nos proporciona una evidencia objetiva de la respuesta inmunológica del huésped contra el cisticerco en el espacio subaracnoideo. Dicho método mide los anticuerpos (AC) contra el cisticerco y tiene la ventaja de reconocerlo en aquellos casos con LCR no inflamatorio. La forma activa de NCC presenta una elevación de inmunoglobulinas (Ig) especificas (IgG, $\operatorname{IgM}, \operatorname{IgE}, \mathrm{y} \operatorname{Ig} \mathrm{A}$ ) en orden decreciente, con los valores más altos detectados entre los casos con quistes intraventriculares o signos inflamatorios en el LCR o aquéllos con manifestaciones clínicas múltiples ${ }^{31}$. La mayor sensibilidad y especificidad se obtiene con el ELISA-IgG (88.5\% y $93.2 \%$ respectivamente $)^{31}$. Los niveles de Ig dependen de la localización del quiste en el $\mathrm{SNC}^{31}$. La sensibilidad en la detección de anticuerpos suele ser mayor en pacientes con quistes múltiples (94\%) $\mathrm{y}$, ostensiblemente menor, en pacientes con quistes únicos o calcificados ${ }^{43}$. Las formas inactivas, granulomatosas y calcificadas no inducen la producción de $\operatorname{IgM}$ y presentan un perfil de LCR similar a los pacientes sin $\mathrm{NCC}^{31}$. Las reacciones falsamente positivas ocurren por una reacción cruzada con otros antígenos parasitarios, como el del Echinococcus o el de la $T$. saginata.

La detección conjunta de IgM e IgG incrementa la sensibilidad del test ${ }^{9}$. La síntesis intracraneal de IgG es una producción local y tiene una correlación estadísticamente significativa con los niveles anticuerpos específicos IgG en $\mathrm{LCR}^{9}$. En cambio, no se ha podido correlacionar ningún test inmunoenzimático particular (IgG, IgM, IgE o IgA) con un estadio evolutivo específico de la larva ni con el número de parásitos ${ }^{31}$. En las formas activas el contenido de Ig es heterogéneo y se relacionado con múltiples factores como la localización del parásito, la inflamación del LCR y la multiplicidad de síntomas clínicos ${ }^{31}$.

A parte de los anticuerpos anti-Taenia específicos que pueden persistir durante mucho tiempo en LCR, la detección de antígenos (Ag) de Taenia puede relacionarse con la fase aguda de la actividad inflamatoria ${ }^{1}$. Si admitimos que la liberación de Ag está relacionada con la actividad inflamatoria y que ésta está relacionada con la actividad clínica de la enfermedad, podemos establecer la hipótesis que la detección de Ag de Taenia con actividad inflamatoria en el LCR se correlaciona con la fase inmunológica activa de la NCC ${ }^{1}$. El Ag de Taenia se detectó en el 47,2\% de los pacientes de Abraham, todos con NCC definitiva, de manera que, se puede considerar un marcador de actividad clínica de la enfermedad ${ }^{1}$.

\subsection{Electroinmunotransfer blot o inmunoblot assay (EITB) para AC de T. solium}

Hasta la introducción del EITB para AC de T. solium, los tests diagnósticos no eran lo suficientemente sensibles ni específicos. Se puede determinar en suero y LCR. Actualmente, con el EITB en LCR se obtiene una sensibilidad diagnóstica del $95-98 \%$ y una especificidad del $100 \%$ en pacientes con quistes múltiples activos intraparenquimatosos o NCC extraparenquimatosa ${ }^{41}$. Un test positivo indica alguna de estas tres posibilidades: teniasis intestinal, NCC o cisticercosis fuera del SNC. De todas formas, hay estudios que demuestran que esta prueba es positiva en sólo el $28 \%$ de los pacientes que tienen una lesión parenquimatosa única y que se puede negativizar cuando el parásito muere ${ }^{16,43}$. En las áreas endémicas la mayoría de seropositivos son asintomáticos ${ }^{37}$.

Se recomienda un seguimiento serológico anual para diferenciar los pacientes curados de los crónicos con lesiones lentamente calcificantes ${ }^{8}$.

\section{Diagnóstico radiológico}

La TC y RM son los métodos de imagen que ofrecen mayor fiabilidad para el diagnóstico. Ante una neuroimagen característica y una serología negativa no se debe excluir la diagnóstico de cisticercosis ${ }^{33}$. La radiología nos ayudará en el momento de establecer un diagnóstico aunque ni la TC ni la RM presentan un $100 \%$ de especificidad para la NCC. La RM es más sensible que la TC, ya que permite diferenciar lesiones peri e intraventriculares, caracterizar mejor las lesiones quísticas y determinar el tratamiento de elección pero, en cambio, es menos sensible para la detección de calcificaciones. Los hallazgos dependen del estadio del ciclo vital del parásito. En el estadio vesicular, la larva aparece como un quiste redondeado, de paredes no visibles y relleno de un líquido isointenso con el LCR con un nódulo mural que es el escólex. Son raros el edema y la captación de contraste. En el estadio granulomatoso, la pared del quiste capta contraste y el líquido intraquístico es hiperintenso. Se pueden encontrar captaciones en anillo en 2/3 de los pacientes y también edema. Esta fase se llama también encefalítica o granulosa. El quiste puede afectar a uno o más lóbulos, con o sin continuidad con el sistema ventricular. En el estadio final (nodular calcificado) la lesión 
reduce su tamaño y está completamente mineralizada, lo cual proporciona una imagen como una diana negra en T2 que refleja las calcificaciones. A veces se observa un punto central hiperintenso que refleja una pequeña cantidad de líquido quístico y no el escólex. El tamaño típico del cisticerco en fase celulosa es de $1 \mathrm{~cm}$, y habitualmente, aunque los quistes pueden alcanzar un gran tamaño, no suelen estar tabicados. Los quistes viables tienen un diámetro de 10-20 mm. A veces se encuentran quistes gigantes que miden mas de $50 \mathrm{~mm}$, siendo más frecuentes en la cisura de Silvio. La encefalitis por cisticerco es una forma rara de la enfermedad y se debe a la presencia de numerosos quistes que conducen a un edema cerebral difuso grave.

La NCC extraparenquimatosa incluye quistes en los ventrículos y cisternas de la base (racemosa). La membrana es fina y el fluido isodenso con el LCR de manera que a veces no son visibles en la TC o ligeramente en la RM. Podemos encontrar hidrocefalia, ependimitis, distorsión de cisternas de la base o meningitis basal.

\section{Tratamiento}

\section{Tratamiento médico}

\subsection{Tratamiento antiparasitario}

Los fármacos antiparasitarios utilizados en el tratamiento de la NCC son el albendazol y el praziquantel. Estudios comparativos han demostrado una mayor efectividad del albendazol en la reducción del número de quistes y la mejoría clínica asociado a una menor tasa de reacciones adversas. El albendazol destruye el $80-90 \%$ de quistes parenquimatosos, mientras que el praziquantel el $60-70 \%{ }^{12}$. Una ventaja adicional del albendazol es su menor coste, por lo que actualmente se considera el medicamento de elección en el tratamiento de la NCC y se recomienda en pacientes sintomáticos con múltiples quistes viables en el parénquima cerebral ${ }^{41}$. El albendazol puede ser útil también en la NCC extraparenquimatosa, especialmente en la forma subaracnoidea racemosa donde la escisión completa de los quistes es casi impracticable. La respuesta es más pobre y se requieren dosis más altas y tratamientos más prolongados ${ }^{2,32}$.

Actualmente existe un intenso debate sobre el valor y seguridad de la terapia anticisticerco. Desafortunadamente, esto ha conducido a la confusión y toma de decisiones poco basadas en la evidencia científica sobre todo en zonas donde la NCC no es un diagnóstico habitual.

La terapia farmacológica no debe ser utilizada de manera indiscriminada en todos los casos, sino individualizada según el síndrome clínico, grado de afectación neurológica, viabilidad de los quistes y respuesta inmune del huésped. El objetivo de la terapia anticisticerco es la destrucción simultánea de múltiples quistes, controlando al mismo tiempo la respuesta inflamatoria con corticoides. Se recomienda la hospitalización del paciente, al menos durante las primeras 72 horas de tratamiento, ya que entre los días 2 y 5 de la terapia se produce una exacerbación de la sintomatología neurológica con la aparición de cefalea, vómitos y crisis en el 50-80\% de pacientes, acompañado de una acentuación de la pleocitosis en LCR en el 50-75\% de casos, debido a la reacción inflamatoria del huésped en respuesta a la muerte del parásito ${ }^{41}$. Estas reacciones se pueden minimizar, e incluso eliminar, aumentando la dosis de corticoides y asociando tratamiento sintomático ${ }^{41}$.

En la reunión de expertos en cisticercosis que tuvo lugar en Lima en 2002 se adoptaron ciertas pautas terapéuticas por consenso ${ }^{17}$ :

\section{- Cisticercosis parenquimatosa:}

- Quistes viables: Consenso en la administración de albendazol $15 \mathrm{mg} / \mathrm{kg}$ durante 8 días o praziquantel $75 \mathrm{mg} / \mathrm{kg}$ dividido en tres dosis tomadas en un día, acompañado de esteroides (ej. dexametasona de 4 a $12 \mathrm{mg} / \mathrm{d}$ ). Hubo controversia en la utilización de antiparasitarios en pacientes con infecciones masivas por el riesgo de efectos secundarios graves.

- Lesiones captantes: La mayoría de miembros no utilizan de manera rutinaria los antiparasitarios en lesiones únicas captantes ya que habitualmente evolucionan bien con el tratamiento anticomicial, independientemente de la administración conjunta de los antiparasitarios, pero algunos consideran que la memoria radiológica justifica su uso. En pacientes con infecciones masivas (encefalitis por cisticerco) hubo consenso en que no se deben utilizar debido a que exacerban la reacción inflamatoria en el parénquima cerebral.

- Calcificaciones. No hay lugar a la utilización de fármacos antiparasitarios pues el parásito ya esta muerto.

\section{- Cisticercosis extraparenquimatosa:}

Dada su asociación a un doble pronóstico, hubo consenso en que debe ser tratada de manera agresiva. Los quistes localizados en el sistema ventricular se deben tratar quirúrgicamente, especialmente si se dispone de endoscopia. Hubo consenso en que la cisticercosis de las cisternas de la base se debe tratar con fármacos antiparasitarios, ya que habitualmente no se puede obtener una resección quirúrgica 
completa y la implantación de un sistema derivativo sólo se asocia a un mal pronóstico. La duración óptima del tratamiento se desconoce, pero debe ser prolongado. Todos los pacientes con cisticercos subaracnoideos se deben tratar también con corticoides. Los quistes gigantes localizados en la cisura de Silvio responden bien a los esteroides en combinación con fármacos antiparasitarios.

Sabemos que en ciertos casos el paciente no responde a la terapia antiparasitaria y se han postulado varias explicaciones. Existe una variabilidad interindividual en las concentraciones plasmáticas de praziquantel y el metabolito activo del albendazol (Asox) ${ }^{27,38,39}$. Existe un factor ligado al sexo -mayor concentración de Asox en mujeres que en hombres- como expresión dominante de isoformas del citocromo $\mathrm{p} 450^{28,29}$. Se han descrito importantes interacciones con alimentos y fármacos. La dexametasona disminuye las concentraciones plasmáticas de praziquantel $\mathrm{y}$ aumenta las de $\mathrm{Asox}^{39,40}$. La dieta rica en carbohidratos, zumo de pomelo y la cimetidina aumentan las concentraciones plasmáticas de praziquantel ${ }^{6,7,25,38,39}$. Las dietas ricas en grasa y la cimetidina pueden también favorecer la absorción del albendazol ${ }^{20}$. Los fármacos antiepilépticos reducen las concentraciones plasmáticas de praziquantel y Asox ${ }^{22}$. Así pues, dada la gran variabilidad interindividual y las interacciones farmacológicas complejas, se recomienda la monitorización de las concentraciones plasmáticas de praziquantel y Asox.

\subsection{Tratamiento anticomicial}

Las formas inactivas (calcificaciones) de la NCC requieren tratamiento crónico con antiepilépticos. Las formas activas de NCC que cursan con crisis también requerirán tratamiento con antiepilépticos. No se conoce la duración de la terapia con fenitoina o carbamacepina, pero se ha sugerido que se debe continuar hasta que los estudios radiológicos seriados muestren la resolución completa de las lesiones agudas y no haya evidencia de calcificaciones. Si se cumplen estos criterios se puede intentar suspender el tratamiento en aquellos pacientes que han permanecido 2 años libres de crisis $^{34}$.

Carpio considera que el riesgo de recidiva tras la primera crisis se puede predecir en función de los cambios radiológicos ${ }^{5}$. Así pues, en su estudio hubo recidiva de las crisis en el 22\% de pacientes en los que el quiste desapareció completamente y en el 56\% de los que persistió lesión activa. En cambio, los pacientes que sufrieron dos crisis presentaron un riesgo del 68\% de hacer una nueva crisis durante los 6 años que siguieron a la segunda crisis. No se halló una disminución de la recurrencia de crisis en aquellos pacientes en que se asoció el tratamiento antiparasitario al anticomicial, res- pecto de aquéllos tratados únicamente con anticomiciales.

\section{Tratamiento quirúrgico}

\subsection{Generalidades}

La cirugía fue el único tratamiento de la NCC hasta el advenimiento de los fármacos antiparasitarios. Actualmente, sus indicaciones están muy restringidas pero todavía en algunos casos es la terapia de elección. Las técnicas quirúrgicas se pueden resumir en tres grupos: cirugía abierta para resección de quistes, implantación de sistemas de derivación ventrículo-peritoneal (DVP) en casos de hidrocefalia y técnicas de endoscopia para escisión de quistes intraventriculares y tratamiento de la hidrocefalia obstructiva.

Se considera que las lesiones quísticas con efecto de masa, clínica de HTIC y déficits neurológicos, son candidatas a cirugía ${ }^{11,26}$. El volumen de la lesión y la clínica de HTIC fue lo que determinó la opción quirúrgica en nuestros casos 1 y 4 . Sin embargo, un estudio reciente de Proaño at al..$^{32}$ presenta una serie de 33 pacientes con quistes subaracnoideos gigantes y con clínica de HTIC, que no requirieron exéresis del quiste. Dichos pacientes fueron tratados con corticoides y antihelmínticos, y sólo fueron intervenidos en caso de hidrocefalia. Cuatro de estos pacientes presentaron secuelas neurológicas, mientras que el resto se recuperaron completamente. Dichos autores recomiendan la exéresis del quiste sólo cuando existe un riesgo vital inminente.

Entre un 15 y un 20\% de las NCC presentan afectación intraventricular ${ }^{30}$. El mayor riesgo de los quistes en esta localización es el desarrollo de hidrocefalia para la cual el tratamiento más frecuente es la DVP. El mayor problema de la implantación de un sistema derivativo en pacientes con NCC intraventricular es la disfunción, generalmente por obstrucción. Se ha descrito una mortalidad de hasta el $50 \%$ a los dos años ligada al numero de intervenciones quirúrgicas por revisión del sistema de derivación ${ }^{10}$.

\subsection{Endoscopia}

La elección de la cirugía abierta o endoscópica depende, en gran parte, de la localización del quiste y de la experiencia del neurocirujano. En caso de quistes de ventrículos laterales y tercer ventrículo, la endoscopia ofrece ventajas respecto a la cirugía abierta. La endoscopia permite extirpar el quiste y, además, realizar una ventriculostomía o septostomía, según el caso, y de este modo nos ofrece la posibilidad de prescindir de una prótesis permanente. Los abordajes ventriculares clásicos, por vía transcallosa o transcortical, se asocian a un mayor riesgo de complicaciones que el abordaje endoscópico ${ }^{4}$. Bergsneider et al. ${ }^{4}$ analizaron 10 
pacientes intervenidos mediante endoscopia, de los cuales, en nueve se consiguió la exéresis completa de las lesiones. Sólo dos pacientes presentaron deterioro postoperatorio y tres de los 10 pacientes requirieron una DVP. Bergsneider et al. ${ }^{3}$ realizaron un abordaje por endoscopia en cinco casos de NCC del IV ventrículo obteniendo en todos los casos la exéresis completa con una mínima morbilidad. En nuestro caso número 6 se optó por el abordaje endoscópico, que nos permitió la resolución de la hidrocefalia sin necesidad de DVP, así como la exéresis completa de la lesión y su diagnóstico definitivo sin morbilidad.

\section{Prevención}

El mejor tratamiento de la cisticercosis es su prevención, de modo que actualmente los esfuerzos se dirigen hacia la creación de vacunas. Una posible solución es la vacunación de cerdos contra la infección por el parásito. Se ha desarrollado una vacuna compuesta de oncosferas de antígenos recombinantes ${ }^{15,23}$. Los antígenos TSOL18 y TSOL45-1A son efectivos induciendo un alto nivel de protección (de hasta el 100\%) ${ }^{15,18,23}$. Esta vacuna tiene el potencial de contribuir al control y a la erradicación de la cisticercosis humana y su coste es bajo ${ }^{15,18,23,24}$.

\section{Conclusiones}

El tratamiento de la NCC debe ser individualizado en cada paciente, en función de la sintomatología, forma y localización. En pacientes con enfermedad inactiva se recomienda únicamente tratamiento sintomático. En la enfermedad activa parenquimatosa no hay datos concluyentes acerca del beneficio del tratamiento con antiparasitarios. Sin embargo, los pacientes con enfermedad activa extraparenquimatosa se pueden beneficiar del tratamiento antihelminítico, asociado a corticoides durante los primeros días. El tratamiento quirúrgico está indicado en lesiones que provocan focalidad neurológica progresiva, HTIC o hidrocefalia. Dado que el mejor tratamiento de la cisticercosis es su prevención, los esfuerzos actuales se dirigen a la creación de vacunas.

\section{Bibliografía}

1. Abraham, R., Pardini, A.X., Vaz, A.D., Livramento, J.A., Machado dos Ramos, L.: Taenia antigens detection in the cerebrospinal fluid of patients with neurocysticercosis and its relationship with clinical activity of the disease. Arq Neuropsyquiatr. 2004; 62 (3B): 756-760.

2. Agapejev, S., Silva, M.D., Ueda, A.K.: Severe forms of neurocysticercosis. Treatment with albendazole. Arq Neuropsyquatr. 1996; 54: 82-93.

3. Bergsneide, M.: Endoscopic removal of cysticercal cysts within the fourth ventricle. Technical note. J Neurosurg. 1999; 91: 340-345.

4. Bergsneider, M., Holly, T.H., Lee, J.H., King, W.A., Frazee, J.G.: Endoscopic management of cysticercal cysts within the lateral and third ventricles. J Neurosurg. 2000; 92: 14-23.

5. Carpio, A., Hauser, W.A.: Prognosis for seizure recurrence in patients with newly diagnosed neurocysticercosis. Neurology. 2002; 59: 1730-1734.

6. Castro, N., Medina, R., Sotelo, J., Jung, H.: Bioavailability of praziquantel increases with concomitant administration of food. Antimicrob Agents Chemother. 2000; 44: 2903-2904.

7. Castro, N., Jung, H., Medina, R., González-Esquivel, D., López, M., Sotelo, J.: Interaction between grapefruit juice and praziquantel in humans. Antimicrob Agents Chemother. 2002; 46: 1614-1616.

8. Cho, S.Y., Kim, S.I., Kang, S.Y.: Serologic follow-up study in neurocysticercosis patients by ELISA after praziquantel therapy. Kisaengchunghak Chapchi. 1986; 24: 159-170.

9. Cho, S.Y., Kim, S.I., Kang, S.Y., Park, A.J.: Intracranial synthesis of specific IgG antibody in cerebrospinal fluid of neurocysticercosis patients. Kisaengchunghak Chapchi. 1988; 26: $15-26$.

10. Colli, B.O., Martelli, N., Assirati, J.A. Jr, Machado, H.R., de Vergueiro Forjaz, S.: Results of surgical treatment of neurocysticercosis in 69 cases. J. Neurosurg. 1986; 65: 309315.

11. Colli, B.O., Martelli, N., Assirati, J.A., et al.: Cysticercosis of the central nervous system. Surgical tratment of cerebral cysticercosis: a 23-year experience in the Hospital das Clinicas of Ribeirao Preto Medical School. Arq Neuropsyquatr. 1994; 52: 166-186.

12. Del Brutto, O.H., Sotelo, J., Roman, G.C.: Neurocysticercosis. A clinical handbook. Swets \& Zeitlinger publishers. Lisse. The Netherlands, 1998. Pp 216.

13. Del Brutto, O.H., Santibáñez, R., Idovro, L., et al.: Epilepsy and neurocysticercosis in Atahualpa: a door-to-door survey in rural coastal Ecuador. Epilepsia. 2005; 46: 583-587.

14. Esquivel, A., Díaz-Otero, F., Giménez-Roldán, S.: Growing frequency of neurocysticercosis in Madrid (Spain). Neurología. 2005; 20: 116-120.

15. Flisser, A., Gauci, C.G., Zoli, A., et al.: Induction of protection against porcine cysticercosis by vaccination with recombinant oncosphere antigens. Infect Immun. 2004; 72: 5292-5297.

16. García, H.H., Gilman, R.H., Catacora, M., Verastegui, M., González, A.E., Tsang, V.C.: Serologic evolution of neurocysticercosis patients after antiparasitic therapy. J Infect Dis. 1997; 175: 486-489.

17. García, H.H., Evans, C.A.W., Nash, T.E., et al.: Current consensus guidelines for treatment of neurocysticercosis. Clinical Microbiol Rev. 2002; 15: 747-756.

18. González, A.E., Gauci, C.G., Barber, D., et al.: Vacci- 
nation of pigs to control human neurocysticercosis. A, J Trop Med Hyg. 2005; 72: 837-839.

19. Hawk, M.W., Shahlaie, K., Kim, K.D., Theis, J.H.: Neurocysticercosis: a review. Surg Neurol. 2005; 63: 123-132

20. Jung, H., Medina, R., Castro, N., Corona, T., Sotelo, J.: Pharmacokinetic of study of praziquantel alone and in combination with cimetidine in a single-day therapeutic regimen. Antimicrob Agents Chemother. 1997; 41: 1256-1259.

21. Kim, I.Y., Kim, T.S., Lee, J.H., Lee, J.K., Jung, S.: Inflammatory aneurysm due to neurocysticercosis. J Clin Neurosc. 2005; 12: 585-588.

22. Lanchote, V.L., García, F.S., Dreossi, S.A., Takayanagui, OM.: Pharmacokinetic interaction between albendazole sulfoxide enantiomers and antiepileptic drugs in patients with neurocysticercosis. Thar Drug Monit. 2002; 24: 338-345.

23. Lightowlers, M.W.: Vaccines for prevention of cysticercosis. Acta Trop. 2003; 87:129-135.

24. Lightowlers, M.W.: Vaccination for the prevention of cysticercosis. Dev Biol. 2004; 119: 361-368.

25. López-Gómez, M., Castro, N., Jung, H., Sotelo, J., Corona, T.: Optimization of the single-day praziquantel therapy for neurocysticercosis. Neurology. 2001; 57: 19291930.

26. Madrazo, I., Proaño, J.V.: Tratamiento quirúrgico de la neurocisticercosis. In: Arriagada C, Nogales-Gaete J, Apt W, eds. Neurocisticercosis. Santiago, Chile: Arryong. 1997; pp. 229-231.

27. Marques, M.P.C., Takayanagui, O.M., Bonato, P.S., Santos, S.R.C.J., Lanchote, V.L.: Enantioselective kinetic disposition of albendazole sulfoxide in patients with neurocysticercosis. Chirality. 1999; 11: 218-223.

28. Marques, M.P., Takayanagui, O.M., Lanchote, V.L.: Albendazole metabolism in patients with neurocysticercosis: antipyrine as a multifunctional marker drug of cytochrome p450. Braz J Med Biol Res. 2002; 35: 261-269.

29. Mirfazaelian, A., Dadashzadeh, S., Rouini, M.R.: Effect of gender in the disposition of albendazole metabolites in humans. Eur J Clin Pharmacol. 2002; 58: 403-408.

30. Obrador, S.: Cysticercosis cerebri. Acta Neurochir. 1962; 10: 320-364.

31. Odashima, N.S., Takayanagui, O.M., de Castro Figuereido, J.F.: Enzyme linked immunosorbent assay (ELISA) for the detection of IgG, IgM, IgE and IgA against cysticercus cellulosae in cerebrospinal fluid of patients with neurocysticercosis. Arq Neuropsyquiatr. 2002; 60 (2B): 400-405.
32. Proaño, J.V., Madrazo, I., Avelar, F., López-Félix, B., Díaz, G., Grijalva, I.: Medical treatment for neurocysticercosis characterized by giant subarachnoid cysts. N Engl J Med. 2001; 345: 879-885.

33. Richards, F., Jr, Schartz, P.M.: Laboratory diagnosis of cysticercosis. Clin Lab Med. 1991; 11:1011-1028.

34. Riley, T., White, A,C.: Management of neurocysticercosis. CNS Drugs. 2003; 17: 577-591.

35. Roca, C., Gascón, J., Font, B., Pujol, T., Valls, M.E., Corachán, M.: Neurocysticercosis and population movements: analysis of 23 imported cases in Spain. Eur J Clin Microbiol Infect Dis. 2003; 22: 382-384.

36. Roman, G.C.: Neurocysticercosis. A public health perspective. Rev Neurol. 2003; 36: 71-74.

37. Sazon, F., Osorio, A.M., Morales, J.P.: Serological screening for cysticercosis in mentally altered individuals. Trop Med Int Health. 2002; 7: 532-538.

38. Schipper, H.G., Koopmans, R.P., Nagy, J., Butter, J.J., Kager, P.A., Van Boxtel, C.J.: Effect of dose increase of cimetidine coadministration on albendazole bioavailability. Am J Trop Med Hyg. 2000; 63: 270-273.

39. Sotelo, J., Jung, H.: Pharmacokinetic optimization of the treatment of neurocysticercosis. Pharmacokinet, 1998; 34: 503-515.

40. Takayanagui, O.M., Lanchote, J.L., Marques, M.P.C., Bonato, P.S.: Therapy for neurocysticercosis: pharmacokinetic interaction of albendazole sulfoxide with dexamethasone. Ther Drug Monit. 1997; 19: 51-55.

41. Takayanagui, O.M.: Therapy for neurocysticercosis. Expert. Rev Neurother. 2004; 4: 129-139.

42. Wallin, M.T., Kurtzke, J.F.: Neurocysticercosis in the United States: review of an important emerging infection. Neurology. 2004: 63: 1559-1564.

43. Wilson, M., Bryan, R.T., Fried, J.A., et al.: Clinical evaluation of cysticercosis enzyme-linked inmunoelectrotransfer blot in patients with neurocysticercosis. J Infect Dis. 1991;164:1007-1009.

Enseñat, J.; Martínez-Mañas, R.; Horcajada, J.P.; De Juan, C.; Ferrer, E.: Dificultades diagnósticas y terapéuticas en la neurocisticercosis: presentacion de 6 casos y revisión de la literatura. Neurocirugía 2007; 18: 101-110.

Correspondencia postal: Rosa M. Martínez-Mañas. Hôpital de Sion. Service de Neurochirurgie. 1950 Sion. Switzerland. 